\title{
Study of a THz IFEL prebuncher for laser-plasma accelerators
}

\author{
C. Sung ${ }^{1}$, S. Ya. Tochitsky ${ }^{1}$, P. Musumeci ${ }^{2}$, J. Ralph $^{1}$, \\ J. B. Rosenzweig ${ }^{2}$, C. Pellegrini ${ }^{2}$, and C. Joshi ${ }^{1}$ \\ Neptune Laboratory, ${ }^{1}$ Department of Electrical Engineering, \\ ${ }^{2}$ Department of Physics, University of California, Los Angeles, California, 90095
}

\begin{abstract}
For monoenergetic acceleration of electrons, the injected particles need to be bunched with the same periodicity as the accelerating structure. In a laser-driven plasma beatwave accelerator, the accelerating structure (plasma wave) is phase-locked to the $\mathrm{CO}_{2}$ beat-wave used to drive it. Using the same beat-wave to generate high power FIR radiation via difference frequency mixing in GaAs ensures that the radiation has the same phase relationship as the plasma wave before it saturates and detunes from the pump. Therefore, this radiation can be used to prebunch an existing electron beam based on an Inverse Free Electron Laser (IFEL) concept. Here we report the progress on the proposed $\mathrm{THz}$ microbunching experiment in the Neptune laboratory. A $50 \mathrm{~cm}$ long prebuncher is optimized using simulation codes for minimum FIR power required. The injected $5 \mathrm{ps}$ long electron beam is expected to form a series of $45 \mu \mathrm{m}$ long microbunches containing over $40 \%$ of the injected current after $1.6 \mathrm{~m}$ drift space following the undulator. Preliminary experimental results on THz generation are also presented.
\end{abstract}

\section{INTRODUCTION}

Due to the limitation of the energy gain gradient and cost efficiency of conventional RF-based accelerators, advanced accelerating concepts, such as laser or electron driven plasma waves based structures, have been proposed as potential solutions for the next generation accelerators and studied worldwide [1]. In recent years, proof-of principle experiments have shown gradients much higher than conventional accelerators [2] in a short distance. However, low quality of the electron beam produced by plasma-based accelerators, for example, the energy spread is continuous, practically limits acceleration to one stage. Therefore, it is of great importance to demonstrate matched injection of an electron beam in a plasma LINAC to produce a high-quality beam potentially suitable for staging.

In the plasma LINAC, in order to obtain monoenergetic acceleration of electrons, the injected particles need to be bunched on a scale shorter than the wavelength of the accelerating structure and with the same periodicity. For a plasma wave resonantly driven at plasma densities $10^{16}-10^{17} \mathrm{~cm}^{-3}$, the plasma wavelength $\lambda_{\mathrm{p}}$ is equal to $340-$ 
$100 \mu \mathrm{m}$, respectively. In order to inject electrons into a narrow phase interval of the plasma wave, an electron beam needs to be prebunched into a series of $\sim 50-15 \mu \mathrm{m}$ long microbunches that separated by the plasma wavelength. An inverse Free Electron Laser (IFEL) technique - an efficient method to produce a train of microbunches at a wavelength as short as $10 \mu \mathrm{m}$ for a stiff relativistic electron beam [3] - can be utilized to modulate an electron beam on the plasma wavelength scale if a suitable high-power FIR source is developed. The comb of $\mathrm{CO}_{2}$ laser lines is an ideal source for generating step tunable radiation in the FIR range of 100-1000 $\mu \mathrm{m}$ through difference frequency generation (DFG) in a nonlinear crystal. Thus there is a strong motivation both to develop a DFG-based source of FIR radiation and to demonstrate microbunching of electrons for laser-plasma accelerators in the $10^{16}-10^{17} \mathrm{~cm}^{-3}$ density range.

For the $\mathrm{CO}_{2}$ laser driven Plasma Beatwave Accelerator (PBWA) experiment at the Neptune laboratory where $\lambda_{\mathrm{p}}=340 \mu \mathrm{m}(\sim 1 \mathrm{THz})$, it was proposed to use a THz IFEL prebuncher for producing short, $50 \mu \mathrm{m}$ electron bunches phase-locked with the accelerating structure [4]. Phase-locking between the electrons and PBWA becomes possible since the same laser beatwave is used to excite the relativistic plasma wave and to generate $\mathrm{THz}$ radiation via DFG in GaAs to drive the IFEL prebuncher.

In this paper, we report on our progress towards the proposed $\mathrm{THz}$ microbunching of electrons. First, we present results of detailed 3-D modeling of IFEL microbunching including beam propagation in a several meter long drifting space following the prebuncher. The optimization of the prebuncher design is carried out for minimizing the required level of $\mathrm{THz}$ power. Second, we report preliminary experimental results on generating $\mathrm{THz}$ radiation in GaAs. It is the first experimental measurements of $\mathrm{THz}$ signals produced by DFG using 300 ps, high-power $\mathrm{CO}_{2}$ laser pulses.

\section{IFEL MICROBUNCHING AT NEPTUNE LABORATORY}

In the PBWA experiment at the Neptune Laboratory at UCLA, two lines of a $\mathrm{CO}_{2}$ laser $(10.6$ and $10.3 \mu \mathrm{m})$ are used to excite a relativistic plasma beat wave $\left(\lambda_{\mathrm{p}}=340\right.$ $\mu \mathrm{m})$ with an acceleration gradient $\sim 1-3 \mathrm{GeV} / \mathrm{m}[5]$.

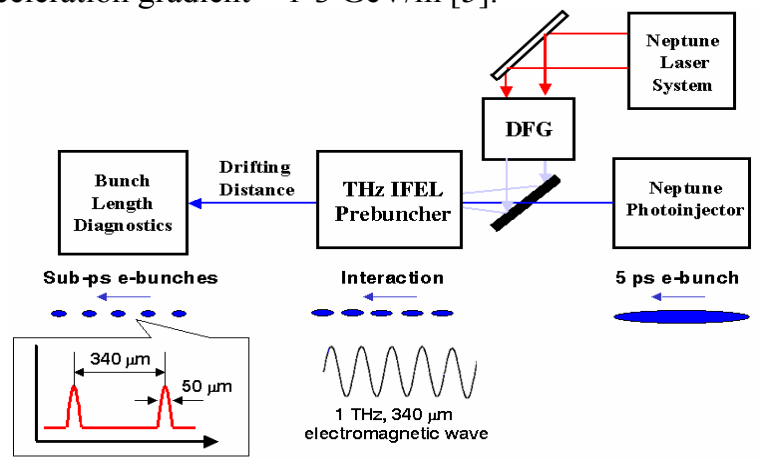

FIGURE 1. Schematic of THz microbunching experiments at Neptune Lab, UCLA 
As shown in Fig. 1, a high-power, two-wavelength $\mathrm{CO}_{2}$ laser beam is sent into a nonlinear crystal (GaAs) for DFG. This newborn radiation will be focused and sent collinearly into a planar IFEL prebuncher with a $10 \mathrm{MeV}$ electron beam coupled through a hole in a mirror. The electron beam coming from a photoinjector will interact with the $\mathrm{THz}$ radiation via IFEL mechanism while going through the undulator and get bunched while drifting in the free space. Since the $340 \mu \mathrm{m}$ wave originates from the same two wavelengths that drive the plasma beat waves, those microbunches potentially can be used for phase-locked injection in the PBWA. After drifting in a vacuum over approximately $1.5-2 \mathrm{~m}$ the bunched beam will be analyzed using different bunch length diagnostics. Several techniques such as OTR/Cherenkov measurements, electro-optical sampling and a RF cavity reflector [6] are considered. It is important that on the $\mathrm{THz}$ scale direct measurement of longitudinal dynamics of electron bunches is still possible.

\section{PHYSICAL PICTURE OF IFEL BUNCHING AND SIMULATION RESULTS}

An IFEL undulator is an array of permanent magnets with their polarization alternatively aligned. When electrons wiggle along the axis of such an undulator, the EM wave can modulate the energy of electrons. Under certain conditions,. Such an energy-modulated beam can be compressed after drifting an optimal distance. If an electron experiences a full cycle of changing of electric field while wiggling one period in the undulator, it is called the resonant condition:

$$
\lambda=\frac{\lambda_{w}}{2 \cdot \gamma^{2}} \cdot\left(1+\frac{K^{2}}{2}\right)
$$

and the undulator dimensionless parameter $K=e B_{w} / m c^{2} k_{w}$, where $\lambda$ is the radiation wavelength, $\gamma$ the electron energy, $\lambda_{\mathrm{w}}$ the undulator wavelength, $\mathrm{k}_{\mathrm{w}}$ the undulator wave number and $B_{w}$ the undulator magnetic field. Under this condition, particles that see the accelerating phase in each wavelength gain energy in the whole wiggling process and those seeing decelerating phases keep losing it. Faster electrons then catch up with slower ones while wiggling in the buncher and drifting in the free space. As a result, microbunches form with the same periodicity as the radiation wavelength. After the optimal bunching position, fast electrons overtake slower ones and debunching occurs.

In a planar IFEL undulator, equations describing the motion of electrons are:

$$
\frac{\partial \psi}{\partial z}=k_{w}+k-\frac{\frac{\partial \gamma}{\partial z}=\frac{K}{\gamma} \cdot \frac{k K_{L}}{\sqrt{1+\frac{\left(z-z_{w}\right)^{2}}{Z_{r}{ }^{2}}}} \cdot \frac{J J(K)}{2} \sin (\psi)}{\left(1-\frac{1+\frac{K^{2}}{2}+\frac{K_{L}}{2}+K K_{L} \cdot J J(K) \cdot \cos (\psi)}{\gamma^{2}}\right)^{1 / 2}}-\frac{1}{Z_{r}\left(1+\frac{\left(z-z_{w}\right)^{2}}{Z_{r}{ }^{2}}\right)}
$$


where $K_{L}$ is the radiation dimensionless parameter $\left(e E_{o} / m c^{2} k\right), \Psi$ is the phase of coupling between the wiggling motion and the EM wave $\left(\Psi=\left(\mathrm{k}+\mathrm{k}_{\mathrm{w}}\right) \mathrm{z}-\mathrm{wt}\right)$, and $\mathrm{JJ}$ is the Bessel factor due to the planar geometry. Those equations show that for a given electron energy $\gamma$ and same radiation power, the larger the $\mathrm{K}$ of the undulator, the stronger the energy modulation is imposed on the electrons.

There are two codes that we use to optimize the undulator design and to model the IFEL bunching effect. The 1-D code solves the equations of motions and calculates the phase and energy of each electron at any longitudinal position. The other is a 3-D code called TREDI that calculates the Lorenz force acting on each electron and calculates its position and energy along time. We first roughly determined parameters of the undulator using the 1-D code and then finalized calculations with the TREDI code. Note that the results obtained by both codes are very similar.

The goal of simulations at this stage is to find the minimum FIR power required to bunch an electron beam with (a) bunch length (FWHM) of less than $50 \mu \mathrm{m}(\lambda \mathrm{p} / 7)$ with a number of trapped particles larger than $40 \%$, and (b) optimal microbunching obtained at a reasonable drifting distance of approximately $1.5-2 \mathrm{~m}$.

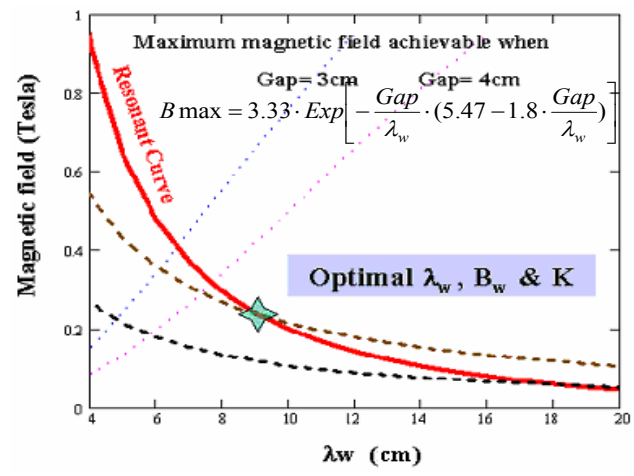

FIGURE 2: Chart for the undulator parameters

Fig. 2 shows the optimization of the undulator parameters. $\lambda_{w}$ and $B_{w}$ are chosen along the solid cure to satisfy the resonant condition and to make $\mathrm{K}$ as large as possible to maximize the bunching effect. However, the Halbach formula limits the choices because of the physical restriction in building magnets with small periods. The gap between the magnets is setup to accommodate the FIR beam inside the undulator. For simplicity, we chose an undulator with $4 \mathrm{~cm}$ gap, $\lambda_{\mathrm{w}}=9 \mathrm{~cm}$, and $\mathrm{B}_{\mathrm{w}}=0.24 \mathrm{~T}(\mathrm{~K}=2)$ for following simulations in a diffraction dominated case.

With those initial undulator parameters, we run 1-D simulations varying the FIR power and the undulator length. The results are shown in Fig. 3. To satisfy conditions (a) and (b), undulator length $\mathrm{L}_{\mathrm{u}}$ can be chosen longer than $50 \mathrm{~cm}$, and the minimum FIR power requirement is around $10 \mathrm{MW}$ when the Rayleigh range is equal to the undulator length. In those cases, the amount of trapped particles is larger than $40 \%$. This results were fully confirmed in 3-D simulations. 

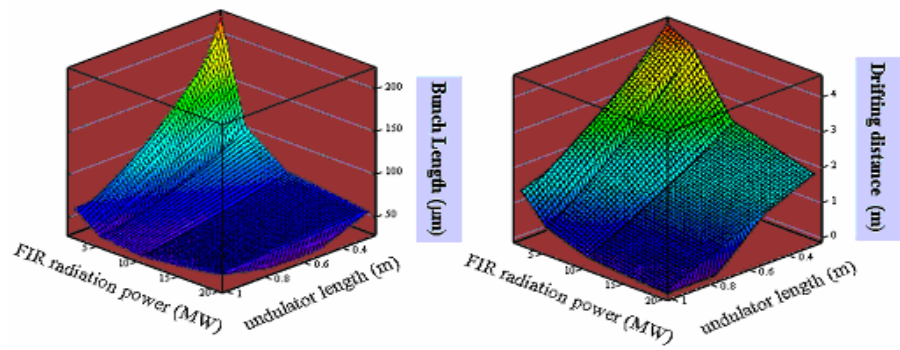

FIGURE 3: 1-D simulation results on bunching with various FIR power and undulator lengths.

(Using an undulator with $4 \mathrm{~cm}$ gap, $\lambda_{\mathrm{w}}=9 \mathrm{~cm}$, and $\mathrm{B}_{\mathrm{w}}=0.24 \mathrm{~T}(\mathrm{~K}=2)$, and $\gamma=20$ )

In Fig. 4, shown is the phase space distribution of electrons in one bucket and its histogram obtained by using the TREDI code. From the histogram, we deduce that the bunch length of $45 \mu \mathrm{m}$ for $40 \%$ trapped particles is obtained at the optimal drifting distance of $1.6 \mathrm{~m}$. Note that the bunch length less than $50 \mu \mathrm{m}$ can be preserved for over $1 \mathrm{~m}$. This large value of the acceptance length is important for future experiments. The undulator, FIR parameters and the optimal bunching results are summarized in Table 1.
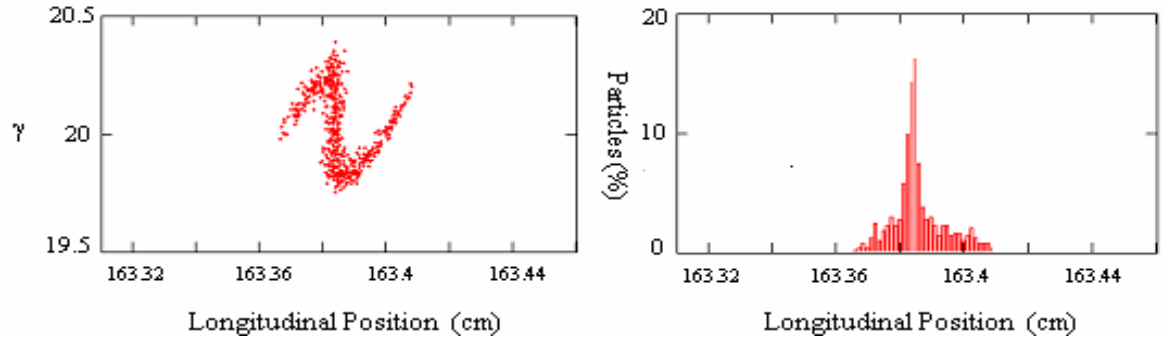

FIGURE 4: Phase space distribution and histogram of electrons in one period of the EM wave. TABLE 1: Important input and output parameters in the optimal bunching case of TREDI simulations

\begin{tabular}{|c|c|}
\hline THz radiation power & $10 \mathrm{MW}\left(\mathrm{I}_{\text {peak }}=5.9 \mathrm{MW} / \mathrm{cm}^{2}\right)$ \\
\hline Injection e energy & $10 \mathrm{MeV}(0.25 \%$ energy spread $)$ \\
\hline Undulator length & $0.5 \mathrm{~m}$ \\
\hline Undulator wavelength & $0.09 \mathrm{~m}$ \\
\hline Permanent magnet & $0.237 \mathrm{~T}$ \\
\hline K factor & 2 \\
\hline Bunch length & $45 \mu \mathbf{m}$ \\
\hline Trapped particles & $\mathbf{4 0 \%}$ \\
\hline Bunching distance & $\mathbf{1 . 6} \mathbf{~ m}$ \\
\hline
\end{tabular}

Thus the values we obtained in simulations are feasible for future experiments: the easy-to-build undulator parameters produced the desired bunch length and 0amount of trapped particles after a reasonable bunching distance. Further decrease in the required FIR power to $\sim 1 \mathrm{MW}$ and less may be possible if $\mathrm{THz}$ beam will be guided in a hollow waveguide with subsequent decrease of the undulator gap to less than $1 \mathrm{~cm}$.

It is interesting that, as shown in [4], the same undulator design can be used to bunch electrons on higher harmonics of the resonant frequency. Therefore, in our case, we can study microbunching of electrons for a plasma density $\sim 10^{17} \mathrm{~cm}^{-3}\left(\lambda_{\mathrm{p}}=114 \mu \mathrm{m}\right)$. 


\section{PRELIMINARY EXPERIMENTAL RESULTS ON THZ RADIATION GENERATION}

We are presently carrying out experiments on generating $\mathrm{THz}$ radiation via $\mathrm{DFG}$ at the Neptune laboratory using high-power, 300 ps laser pulses. The $10.6 \mu \mathrm{m}$ and 10.3 $\mu \mathrm{m} \mathrm{CO} 2$ laser beams were sent on a GaAs crystal according to a noncollinear phasematching configuration [4,7]. The optical scheme of the experiment is shown in Fig. 5.

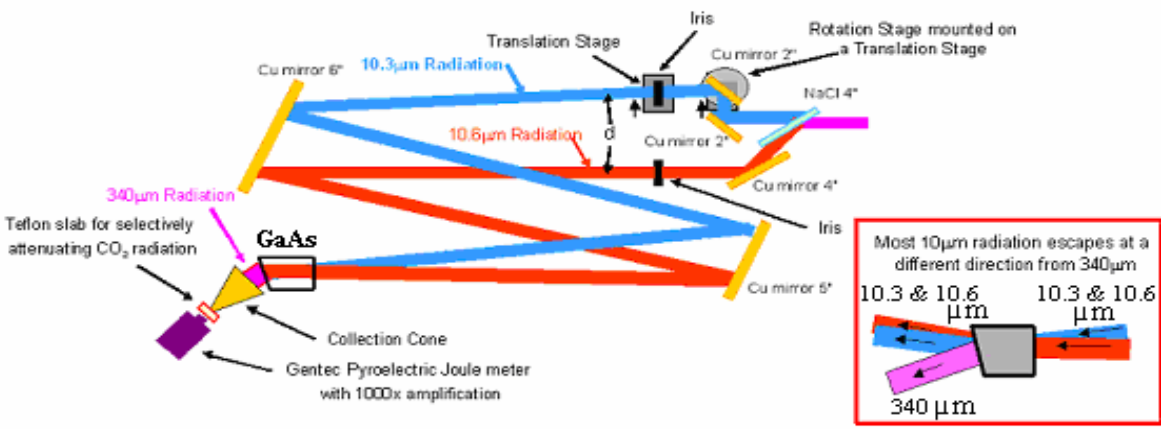

Figure 5. Optical scheme for the THz generation in GaAs

Here a vertically polarized, two-wavelength $\mathrm{CO}_{2}$ laser beam is split into two optical arms. Because of the orientation of the crystal, only one wavelength in each arm is participating in difference frequency mixing. The $10.6 \mu \mathrm{m}$ arm is aligned at a normal to the front surface of the crystal. The $10.3 \mathrm{arm}$ is adjusted to scan the internal phase matching angle. A $2 \times 4 \times 2.5 \mathrm{~cm}$ GaAs crystal is cut in such a way that the newborn radiation is separated from the pump lasers due to refraction at the crystal to air interface (see the insert in Fig. 5). The THz radiation is collected by a cone and sent through a Teflon slab $(10 \mu \mathrm{m}$ radiation attenuator) into a Gentec detector equipped with a $1000 \mathrm{X}$ amplifier. For a very long base of both arms, we achieve an angle resolution of less than $0.01^{\circ}$ while scanning the phase matching angle. The preliminary data on $\mathrm{THz}$ generation is shown in Fig. 6.

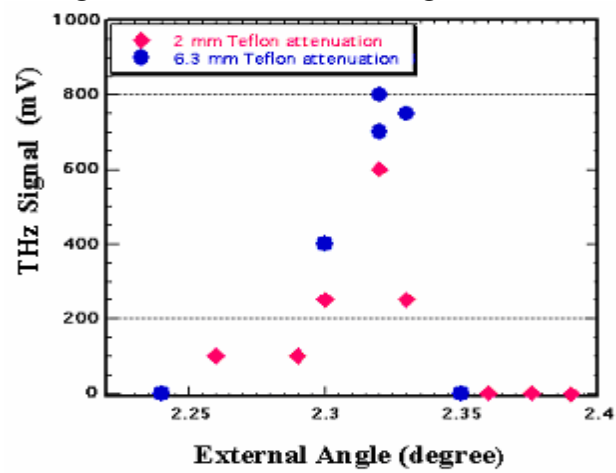

FIGURE 6. Phase matching angle dependence for $\mathrm{THz}$ generation 
In Fig. 6, we can see the angular dependence of measured $\mathrm{THz}$ signals. The phase matching angle corresponded to the peak signal and the angle width are consistent with the theoretical predications for a $2.5 \mathrm{~cm}$ long crystal. Similar signals obtained with different attenuation points that there is no residual $10 \mu \mathrm{m}$ radiation. Assuming flat spectral response of a Gentec energy meter from 1 to $28 \mathrm{THz}$, at least $40 \mathrm{~kW}$ of FIR was generated in GaAs. For the best shots up to $\sim 1.25 \mathrm{~J}(300 \mathrm{~mJ}$ at $10.6 \mu \mathrm{m}$ and $200 \mathrm{~mJ}$ at $10.3 \mu \mathrm{m}$ participated in DFG) was sent on the crystal.

It is known that nonlinear frequency conversion for a pulsed beam is limited by the surface damage threshold of the material. In a series of damage threshold measurements, we observed a single shot damage of GaAs at $\sim 0.5 / \mathrm{cm}^{2}\left(\sim 1.6 \mathrm{GW} / \mathrm{cm}^{2}\right)$. However, in the experiment for a $2 \mathrm{~cm}$ beam, existence of the hot spots seriously limited the incident fluence especially for multishot exposure. We believe that a large aperture ( $\sim$ ") crystal and a corresponding $\mathrm{CO}_{2}$ laser beam of high-optical homogeneity are needed to achieve $\mathrm{THz}$ power in multi-megawatt range without damaging crystal. This is the subject of future work.

\section{CONCLUSIONS}

In this paper, feasibility of prebunching an electron beam for laser-plasma accelerators with various densities has been studied. An undulator design is optimized for the strongest bunching. According to simulations, a bunch length less than $50 \mu \mathrm{m}$ can be achieved with $\mathrm{a} \sim 6 \mathrm{MW} / \mathrm{cm}^{2}$ level intensity of $\mathrm{THz}$ radiation. Preliminary experimental results confirm that DFG can be used to provide such FIR radiation, however, further improvements and studies are needed, such as improving the pump beam profiles, optimizing the phase-matching scheme, guiding of $\mathrm{THz}$ radiation, or adding a chicane to shorten the drifting distance.

\section{ACKNOWLEDGMENTS}

This research was supported by the U.S. Department of Energy under Contract No. DE-FG03-92ER40727.

\section{REFERENCES}

1. T. Tajima and J. M. Dawson, Phys. Rev. Lett., vol. 43, pp. 267-270 (1979)

2. E. Esarey, P. Sprangle, J. Krall, and A. Ting, IEEE Trans. on Plasma Science, vol. 24, pp. 252-288 (1996)

3. W. D. Kimura, M. Babzien, I. Ben-Zvi, et al, Phys. Rev. Lett., vol. 92, 054801-1-054801-4

4. S. Ya. Tochitsky, P. Musumeci, C. E. Clayton, AAC Proceeding 2002, pp. 786-795

5. S. Ya. Tochitsky, R. Narang, C. V. Filip, et al. Phys. Rev. Lett., vol. 92, 095004-1-095004-4 (2004)

6. R. J. England, et al, in this proceeding

7. R.L Aggarwal, B. Lax, and G. Favrot, Appl. Phys. Lett., vol. 22, pp. 329-330 (1973) 